\title{
"Universe collapse model" and its roles in the unification of four fundamental forces and the origin and the evolution of the universe
}

\author{
Jiapei Dai \\ Wuhan Institute for Neuroscience and Neuroengineering, South-Central University for Nationalities, Wuhan, China; \\ jdai@mail.scuec.edu.cn, jiapeidai@msn.com
}

Received 3 March 2012; revised 10 April 2012; accepted 20 April 2012

\begin{abstract}
To unify the four known fundamental forces and provide an explanation for the origin and the evolution of the universe are two long-term goals of theoretical physics. Here a "universe collapse model" has been proposed. The universe consists of Matter and No-matter. Nomatter is the universal energy that constructs a consistent universe field, presenting a spiral wave motion at the speed of light at the small scale. The partial collapse of the universal energy forms the particles of the universal energy in a variety of sizes, which are called as the elementary particles. These elementary particles form atom and matter, which construct the galaxies. The collapse of the universe field induces the formation of the universe collapse potential (UCP) and universe collapse force (UCF), and the later is represented by four different aspects of the fundamental forces at the large or small scales. The mathematical equation and the derivation of UCP and UCF are described, and possible experimental tests are also suggested. Therefore, this new model may give a novel explanation for the unification of four fundamental forces and the origin and the evolution of the universe.
\end{abstract}

Keywords: Universe Collapse Model; Field Theories; Beyond Standard Model; String Field Theory; Grand Unification Model; Fundamental Forces; Elementary Particles; The Origin and Evolution of Universe

\section{INTRODUCTION}

A long-term goal of theoretical physics is to develop a theory or model, which can unify the four known funda- mental forces (or interactions) and give an explanation for the origin and the evolution of the universe, and finally step toward a true theory of everything or reach the ultimate goal of the Grand Unification Model. Up to now, the most promising models are the Standard Model, the String theory and the Big Bang theory as well as their extensions, revisions or replacements.

The Standard Model is a theory that has been proposed to unify the electromagnetic force, the strong force and the weak force with the exception of the gravity [1-5]. Because of its success in explaining a wide variety of experimental results, the Standard Model is widely accepted in particle physics [6-10]. However, its deficiencies that are inconsistent with general relativity, and give no explanation of the origin of mass, the strong $\mathrm{CP}$ problem, the neutrino oscillations, the matter-antimatter asymmetry and the nature of dark matter and dark energy, and in particular that the Higgs particle (also called the God particle) has not yet been discovered, allow us to raise doubt on the Standard Model and related theories [11-13].

The String theory is proposed in order to correct for these deficiencies mentioned above. Although String theory including its extensions such as M-theory allows for the consistent combination of quantum field theory and general relativity, agrees with general insights in quantum gravity such as the holographic principle and black hole thermodynamics, and has been considered as a complete theory of the universe [14-19], however, it has also been criticized for not providing any quantitative experimental predictions such as the very high energies needed to test quantum gravity, the lack of uniqueness of predictions due to the large number of solutions, and the lack of background independence and a full mathematical description [20-22]. In addition, the recent findings disfavor current models of quantum gravity [23].

The Big Bang theory is the prevailing cosmological model that explains the early development of the uni- 
verse and provides the most accurate and comprehensive explanation for the full range of phenomena observed $[24,25]$. However, some problems with the Big Bang theory are existed such as the horizon problem, the flatness/oldness problem, the baryon asymmetry, the globular cluster age and the nature of dark matter and dark energy [26-31]. Therefore, to construct a theory that could provide a satisfying explanation for all existing questions is still a great challenge of theoretical physics.

\section{THE UNIVERSE COLLAPSE MODEL}

Here I propose a new model, called "the universe collapse model”, and the basic principles of this model are as follows:

1) The universe consists of two components: Matter and No-matter. No-matter is proposed as the universal energy. The dark energy recently hypothesized in the universe may be a type of no-matter.

2) The universal energy forms a consistent energy field, i.e., a "universe field", whose distribution may be asymmetric. The universe field is also called the first universe and presents the spiral wave motion at the speed of light at the small scale, but it moves in a large circular or a repeating manner at the large scale, forming a flat spheroid, which is the shape of the whole universe. The motion of the universe field partially complies with Einstein's theory of relativity.

3) The partial collapse and the fracture of the universal energy form the universal matter. The collapsing process of the universe field may be due to the notable reduction of the wave motion speed of the universe field or the fracture of the universe field, for example, a process of partial universe explosion. As the result, the consistent universal energy is mangled and becomes the energy particles of various sizes. These energy particles are the elementary particles of the universal matter, and they have been able to form atoms and construct the galaxies, which are called the "second universe". It is also understandable that such energy particles can transform between themselves via separation and merger.

4) The collapse of the universe field forms not only the universal matter, but also the universe collapse potential (UCP) and the universe collapse force (UCF) due to the interaction and the transformation between the universal matter and the universe field, which means that the UCP and the UCF could be formed around any matters from an elementary particle to a galaxy.

The UCP depends on the degree and the size of the collapse or the characteristic (mass) of a matter such as an elementary particle, a planet, a star or a galaxy. The equation form and the derivation of UCP and UCF are described as follows:

The spiral wave-like motion speed of the universe filed is proposed to be the same as the speed of light (C). The notable decrease of the speed of the universe field from $\mathrm{C}$ to $\mathrm{V} i$ causes the matter to form in the universe, called the universe energy to matter transformation. At the same time, the UCP and UCF are also formed.

Since the most of collapse universe energy is transformed to Mass and the remaining part is to UCP, which mediates the interactions between the mater and the universe field. Therefore, according to the law of conservation of energy, the partial change of collapse universe energy is transformed to UCP, and the equation can be written as:

$$
\mathbf{U C P}=\mathbf{E 1}-\mathbf{E 0}
$$

According to Einstein's mass-energy equation

$$
\mathbf{E 0}=\mathrm{MC}^{\wedge} 2
$$

where the $\mathrm{M}$ is the mass of a matter such as the Sun or an elementary particle.

$\mathbf{E 1}$ is the sum of the whole energy of a matter (here is referred to the energy of motion) and can be defined as:

$$
\begin{aligned}
\mathbf{E 1} & =2 / 1 \mathrm{MV} 1 \wedge 2+2 / 1 \mathrm{MV} 2 \wedge 2+2 / 1 \mathrm{MV}^{\wedge} \wedge 2 \cdots 2 / 1 \mathrm{MVn}^{\wedge} 2 \\
& =2 / 1 \mathrm{M} \sum_{i=1}^{n} \mathrm{Vi}^{\wedge} 2
\end{aligned}
$$

$\sum_{i=1}^{n} \mathrm{Vi} \wedge 2$ is the sum of the squares of the speeds of a matter movement, which includes the speeds of galaxy (V1, revolution speed), star (V2, V3, revolution and spin speeds, respectively) and planet (V4, V5, revolution and spin speeds, respectively), etc, in which a matter exists.

Therefore,

$$
\begin{aligned}
\mathbf{U C P} & =2 / 1 \mathrm{M} \sum_{i=1}^{n} \mathrm{Vi}^{\wedge} 2-\mathrm{MC}^{\wedge} 2 \\
& =\mathrm{M}\left(2 / 1 \sum_{i=1}^{n} \mathrm{~V} i \wedge 2-\mathrm{C}^{\wedge} 2\right)
\end{aligned}
$$

The UCP is a vector unit, and it depends on the matter motion feature.

The point UCP can be defined as:

$$
\begin{aligned}
\mathbf{U C P}(\mathbf{p}) & =\mathrm{UCP} / \mathrm{r} \wedge 2 \\
& =\mathrm{M}\left(2 / 1 \sum_{i=1}^{n} \mathrm{Vi} \wedge 2-\mathrm{C}^{\wedge} 2\right) / \mathrm{r} \wedge 2
\end{aligned}
$$

$r$ is the distance from the matter.

Therefore, the UCF for a matter (m), which is located within the UCP formatted by other matter $(\mathrm{M})$, can be expressed as follows:

$$
\mathbf{U C F}=\operatorname{mUCP}(\mathbf{p})
$$

Therefore, 


$$
\mathbf{U C F}=\operatorname{mM}\left(2 / 1 \sum_{i=1}^{n} \mathrm{Vi}^{\wedge} 2-\mathrm{C}^{\wedge} 2\right) / \mathrm{r}^{\wedge} 2
$$

The UCP of each matter can interact with each other via an interaction force. The interactions between the matters could results in the attractive or repulsive effects depending on the vector direction of UCP of each matter.

5) The four known fundamental forces are different aspects of the UCF at the large or small scales.

\section{THE UNIVERSAL GRAVITATION}

The nature of the universal gravitation is one aspect of the UCF, which is due to the interaction between the matter and the universe field at the large scale. The matter acts on the universe field around itself and results in UCP; in contrast, the universe field acts on the matter and influences the behaviors of the matter such as the characteristics of the matter movement. In addition, the UCP could restrict the matters such as a planet or a galaxy in the certain space, and maintain their relative stability and influence their evolution and fate.

The movement and the revolution patterns of the planets could be affected by UCF. For example, the revolution pattern of a planet in the solar system is related to the UCF due to the evolution of the sun. It is reasonable to say that Newton's universal gravitation (each body in the universe was attracted toward every other body by a force) may be not appropriately defined, and it is just a type of UCF. Therefore, Newton's gravity equation that is consistent with the equation form of UCF described above is only suitable for the solar system, and needs to be modified out of the solar system.

In addition, the precession of the planets in solar system may be due to the gradual reduction of Sun's UCP because of the gradual lost of Sun's mass.

It may also support an explanation for the grotesquery of some galaxies found in the universe, which present "migration" movement because of the obvious change of the UCP of galaxies due to the unique characteristics of these galaxies such as the obvious changes in size and mass. Such kind of motion pattern of a galaxy could not be explained by Newton's law of universal gravitation.

\section{ELECTROMAGNETIC FORCE, STRONG FORCE AND WEAK FORCE}

According to the universe collapse model, the movement of any elementary particles including the electron could form the UCP at the small scale level (micro-level) due to their interactions with the universe field. The chaos movement of the elementary particles may present an undetected UCF because they eliminate each other, however, the ordered movement of the particles, for example, the movement of the electrons along the conduc- tor materials, could form an obvious directional UCF, which can be considered as the electromagnetic force, the strong force and weak force at different situations, depending on how the elementary particles interact with the universe field, and the movement way of each particle as well as the relationships between themselves. Such interactions could be attractive or repulsive, allowing the elementary particles to assemble or disperse, and conesquently, to form different kinds of atoms, which show unique characteristics such as the atom radiation.

\section{THE NATURE OF THE ELECTROMAGNETIC WAVE}

It is necessary to reconsider the nature of the electromagnetic wave such as lights. According to the universe collapse model, the electromagnetic waves are the energy particles, and they can spread along the universe field (the first universe). Because of the spiral wave-like motion of the universe field at the speed of light, the emitted light particles or the electromagnetic particles from the matter can be carried by the universe field, which consequently presents a wave-like movement at the speed of light. In other words, the electromagnetic waves are the energy particles after the emission from a matter in the second universe, but they present a wave-like movement in the first universe. This characteristic is known as the wave-particle duality of electromagnetic waves according to quantum theory. Because the wave motion of the universe field complies with Einstein's theory of relativity at the speed of light, the wave-particle duality of electromagnetic waves (and other elementary particles) is just the type of movement of the energy particles along the spiral wave motion universe field. The curve of light when it passes by the sun (also for a galaxy or a star) is due to the existence of the sun's UCP, which is consistent with Einstein's theory of relativity.

\section{THE INTERACTION BETWEEN THE UNIVERSE MATTER AND NO-MATTER RELATED TO THE ORIGIN AND EVOLUTION OF THE UNIVERSE}

The origin and the evolution of the whole universe may be due to the interaction and the transformation between the universe matter and no-matter (the first universe and the second universe). This is a circular or repeating process that may also be responsible for the origin and the fate of a galaxy, a star or a planet. Such a proposed figuration of the whole universe including the first and the second universes could argue against the Big Bang theory and suggests that a singularity may not exist for the origin of the whole universe, but it could be true for the origin of a galaxy. In addition, it can be suggested that a black hole is just the beginning of a new star, 
therefore, the speed of the energy particles in a black hole are tendency to "very small", and result in a huge strong UCP or UCF according to the equation form described above. The fate of a star is due to the lost of mass, which is transferred to the consistent universe filed. The electromagnetic waves such as photons are the mediators for the interaction and transformation between the universe matter and the universe filed.

\section{THE POSSIBLE EXPERIMENTAL TESTS}

The key point of the universe collapse model is the proposed universe field (first universe), which presents the spiral wave motion at the speed of light at the small scale, but it moves in a large circular or a repeating manner at the large scale. Such a proposal means that the motion of universe field has direction and speed at the large scale, but its spiral wave motion at the speed of light at the small scale is random. A relatively simple experiment is to observe "Brownian motion" (random drifting of particles suspended in a fluid or a gas) at the International Space Station (zero gravity), and consequently could establish whether the Brownian motion for a group of particles (matter) such as a drop of ink in water could have a clear direction besides the random distribution, called directional Brownian motion. The speed of such a directional Brownian motion may indicate the speed of universe field at the large scale.

\section{REMARKS AND CONCLUSIONS}

In this model, the three key basic points are described: 1) The universe field (first universe) presents the wave motion at the speed of light at the small scale; 2) the collapse of universe field forms energy particles (elementary particles), which construct the universe matter (second universe); 3) the interaction and transformation between the universe field and the universe matter may reflect the basic laws and phenomena of the universe evolution.

In addition, the chemical reactions, biological phenomena as well as earth's behavioral changes such as earthquake and natural disasters may be due to the interactions between the matter and no-matter.

\section{ACKNOWLEDGEMENTS}

This work was supported by the Research Foundation from SouthCentral University for Nationalities (XJS09001).

\section{REFERENCES}

[1] Glashow, S.L. (1961) Partial-symmetries of weak interacttions. Nuclear Physics, 22, 579-588. doi:10.1016/0029-5582(61)90469-2
[2] Weinberg, S.A. (1967) Model of leptons. Physical Review Letters, 19, 1264-1266. doi:10.1103/PhysRevLett.19.1264

[3] Englert, F. and Brout, R. (1964) Broken symmetry and the mass of gauge vector mesons. Physical Review Letters, 13, 321-323. doi:10.1103/PhysRevLett.13.321

[4] Higgs, P.W. (1964) Broken symmetries and the masses of gauge bosons. Physical Review Letters, 13, 508-509. doi:10.1103/PhysRevLett.13.508

[5] Guralnik, G.S., Hagen, C.R. and Kibble, T.W.B. (1964) Global conservation laws and massless particles. Physical Review Letters, 13, 585-587. doi:10.1103/PhysRevLett.13.585

[6] Hasert, F.J., Faissner, H., Krenz, W., Von Krogh, J., Lanske, D., et al. (1973) Search for elastic muon-neutrino electron scattering. Physics Letters B, 46, 121-124. doi:10.1016/0370-2693(73)90494-2

[7] Hasert,F.J., Faissner, H., Krenz, W., Von Krogh, J., Lanske, D., et al. (1973) Observation of neutrino-like interactions without muon or electron in the gargamelle neutrino experiment. Physics Letters B, 46, 138-140. doi:10.1016/0370-2693(73)90499-1

[8] Hasert, F.J., Faissner, H., Krenz, W., Von Krogh, J., Lanske, D., et al. (1973) Observation of neutrino-like interactions without muon or electron in the Gargamelle neutrino experiment. Nuclear Physics B, 73, 1-22. doi:10.1016/0550-3213(74)90038-8

[9] Weinberg, S. (2004) The making of the standard model. European Physical Journal C, 34, 5-13. doi:10.1140/epjc/s2004-01761-1

[10] T'Hooft, G. (2007) The making of the Standard Model. Nature, 446, 271-273. doi:10.1038/446271a

[11] Waldrop, M.M. (2011) Unification+150, Nature, 471, 286288. doi:10.1038/471286a

[12] Collision Course. (2011) What will scientists do if they fail to find the Higgs boson? Nature, 479, 6 .

[13] Brumfiel, G. (2011) Higgs hunt enters endgame. Nature, 479, 456-457. doi:10.1038/479456a

[14] Green, M.B., Schwarz, J.H. and Witten, E. (1987) Superstring theory. Cambridge University Press, Cambridge.

[15] Polchinski, J. (1998) String theory. Cambridge University Press, Cambridge.

[16] Becker, K., Becker, M. and Schwarz, J.H. (2007) String theory and M-theory: A modern introduction. Cambridge University Press, Cambridge.

[17] Kiritsis, E. (2007) String theory in a nutshell. Princeton University Press, Princeton.

[18] Brumfiel, G. (2006) Our universe: Outrageous fortune. Nature, 439, 10-12. doi:10.1038/439010a

[19] Berman, D.S. (2008) M-theory branes and their interactions. Physics Reports, 456, 89-126. doi:10.1016/j.physrep.2007.10.002

[20] Damour, T. and Nicolai, H. (2008) Symmetries, singularities and the De-Emergence of space. International Journal of Modern Physics D, 17, 525-531. doi:10.1142/S0218271808012206 
[21] Berkooz, M. and Reichmann, D. (2007) A Short review of time dependent solutions and space-like singularities in string theory. arXiv:0705.2146 [hep-th].

[22] Rozali, M. (2008) Comments on background independence and gauge redundancies. arXiv:0809.3962

[23] Abdo, A., et al. (2009) A limit on the variation of the speed of light arising from quantum gravity effects. $\mathrm{Na}$ ture, 462, 331-334. doi:10.1038/nature08574

[24] Peebles, P.J.E., Schramm, D. N., Turner E. L. and Kron, R.G. (1991) The case for the relativistic hot big bang cosmology. Nature, 352, 769-776. doi:10.1038/352769a0

[25] Khoury, J., Ovrut, B.A., Steinhardt P.J. and Turok, N. (2001) Ekpyrotic universe: Colliding branes and the origin of the hot big bang. Physical Review D, 64, 123522. doi:10.1103/PhysRevD.64.123522

[26] Chung, D.J.H. and Freese, K. (2000) Cosmological challenges in theories with extra dimensions and remarks on the horizon problem. Physical Review D, 61, 2351.

[27] Turner, M.S. and Weinberg, E.J. (1997) Pre-big-bang inflation requires fine-tuning. Physical Review D, 56, 46044609. doi:10.1103/PhysRevD.56.4604

[28] Gott, J.R. (1982) Creation of open universes from de Sitter space. Nature, 295, 304-307. doi:10.1038/295304a0

[29] Farrar, G.R. and Zaharijas, G. (2006) Dark matter and the baryon asymmetry of the universe. Physical Review Letters, 96, 041302. doi:10.1103/PhysRevLett.96.041302

[30] Pont, F., Mayor, M., Turon, C. and Vandenberg, D.A. (1998) Hipparcos subdwarfs and globular cluster ages: The distance and age of M 92. Astronomy and Astrophysics, 329, 87-100.

[31] Ratra, B. and Vogeley, M.S. (2008) The beginning and evolution of the universe. Publications of the Astronomical Society of the Pacific, 120, 235-265.

doi:10.1086/529495 